\title{
"A Tensorial Form of the Theory of Functions". An Engineering Application to: Polynomial Interpolation
}

\author{
J.L. Urrutia-Galicia \\ Coordinación de M ecánica A plicada \\ Instituto de Ingeniería, UNAM
}

(recibido: febrero de 2004; aceptado: agosto de 2004)

\begin{abstract}
From basicconcepts such as: ten sor cal cu lus (Flügge, 1972); functional anal y sis (M ikhlin, 1964) and sol id mechan ics (Soedd, 1972) theob jectiveof yhis objetiveis to show that besides the " $n$ " covariant functions (of functional anal $y$ sis), lin early in depend ent and notneces sar ily or thogonal, thereis another group of " $n$ " contravariant func tions that are biorthogonal to the for mer group. The pre sen ta tion of these twofami lies gives riseto a new for mu la tion of functional anal y sisin skew co or di nates. Wewill seeth at the con cept of skew man i foldsfindsim medi ateap pli ca bil ity totheproblem of in ter pola tion ofar bi trary func tions via the use of thenew con cept of covariant and contravariant poly no mi als. Thetheory and theex am ples demon stratethat the prob lems of in ter po la tion and Fou rier anal y siscan begrouped into onesin gletheory.
\end{abstract}

Keywords: In ter po la tion, in dex nota tion, covariant and contravariant poly no mi als, gen eral skew man i folds (Ten sor cal cu lus), ten sorial theory of functions, con ver gence.

\section{Resumen}

A partir de conceptos básicos de cálculo tensorial (Flügge, 1972), análisis funcional (Mikhlin, 1964) y de mecánica de sólidos (Soedel, 1972), el objetivo de este artículo es demostrar que además de las " $n$ " funciones covariantes (de análisis funcional), linealmente independientes pero no necesariamente ortogonales, existe otro grupo de " $n$ " funciones contravariantes que son biortogonales al grupo ante rior. La presentación de estas dos familias de funciones da origen a una nueva formulación de análisis funcional en coordenadas oblicuas. Veremos que el concepto de espacios coordenados oblicuos encuentra aplicación inmediata al problema de interpolación de funciones arbitrarias vía el uso del nuevo concepto de polinomios covariantes y contravariantes. La teoría y los ejemplos demuestran que los problemas de interpolación y análisis de Fourier se pueden agrupar y tratar dentro de una sola y única teoría.

Descriptores: Interpolación, notación índice, polinomios covariantes y contravariantes, espacios gener ales oblicuos (cálculo tensorial), teoría tensorial de funciones, convergencia.

\section{Introduction}

One of the most controversial topics in numerical analysis is the problem of interpolation and a great variety of approximate methods can be found. However, when we exam ine "Why and in what sense are those methods accurate" we find a disenchan- ting panorama since there are no answers to those ques tions (Carnaham et al., 1969) and (Forsythe et al., 1977). When try ing to ap proxi mate a given arbitraryfunc tionf $(\mathrm{x})$ with some poly no mial

$$
f(x)=\sum_{n=0}^{N} a_{n} x^{n}
$$


it is a common procedure to selectn +1 points and to $o b$ tain the $a_{n} c o$ efficients from the so lution of the following $n+1$ equations

$$
\begin{gathered}
a_{0}+a_{1} x_{0}^{1}+a_{2} x_{0}^{2}+a_{3} x_{0}^{3}+\ldots+a_{n} x_{0}^{n}=f\left(x_{0}\right) \\
a_{0}+a_{1} x_{1}^{1}+a_{2} x_{1}^{2}+a_{3} x_{1}^{3}+\ldots+a_{n} x_{1}^{n}=f\left(x_{1}\right) \\
\ldots \ldots \ldots \ldots \\
a_{0}+a_{1} x_{n}^{1}+a_{2} x_{n}^{2}+a_{3} x_{n}^{3}+\ldots+a_{n} x_{n}^{n}=f\left(x_{n}\right)
\end{gathered}
$$

It is clear that the choice of the $n+1$ points is not unique, and de fin ing which group is the best is a tremendous task. There are a great number of possible sets of points to be selected. However, we can not decide conclusively from which group of points we can get our best ap proxi ma tion tof(x). Quite easily we come across statements like (Forsythe et al., 1977) "The cri te rion of rea son ableness (of a given polynomial approximation to a function $f(x)$ ) may vary from problem to problem and may neverbe sat is fac to rily un der stoo $d "$ "When we deal with measured or tabulated values of a function $f(x)$ that depends on $x$, one possible approach could be the method of di vided dif fer ences of Newton. Unfortunately, the same doubts arise with re spect to the ap proxi ma tion and the sense of con vergence of the pro posed in terpo la tions.

In experimental analysis, it is usual to cull experimentalvalues $f_{i}(x)$ and val ues of the exper imental variable $x_{i}$. The problem is to find (Fraleigh and Beauregard, 1990) some function $f(x)=r_{0}+r_{1} x$ with certain values of $r_{0}$ and $r_{1}$ that fits accurately our exper i ments. How ever, no men tion is made of the sense and rate of con ver gence of the func tion $f(x)$ ob tained. We only note that some how our func tion ap proaches very closely our data points $f_{i}(x)$.

Maybe one of the most pop u lar meth ods is the one pro posed by Lagrange. It of fers the pos si bil ity of getting one special polyno mial that repro duces exactly each and every data. However the same doubt arises regarding exactness of our approxi ma tion. At this point it has to be noted that, one major drawback of other methods is the handling of se quences like, $\left(1, x, x^{2}, x^{3}, \ldots, x^{n}\right)$ notor thogo nal among them by using the Gram-Schmidt orthogonalization procedure in an attempt to get sim plic ity. In view of this, it is not sur pris ing that in manyprob lems of interpolation we resort to or thogonal polynomials like those of Laguerre, Chebyshev or Legendre among many others. The reason for this choice is, ap par ently, a better con ver gence. How ever, no clear def i ni tions of con ver gence are pro vided.

Searching for some clues to the con ver gence of someinterpolating polynomial we find the following Faber's The o rem (Forsythe et al ., 1977):

"For any in ter po lat ing array thereex ists a con tin $u$ ous function $g$ and an $x$ in $[a, b]$ such that $P n(g)(x)$ does not con verge to $g(x)$, as $n \rightarrow \infty "$.

An example of this problem of divergence is Runge's Func tion pre sented in ref er ence (Forsythe et al., 1977).

Up to this point we have been speak ing of in terpolation with orthogonal (Legendre) and with nonorthogonal functions via different methods with out men tion ing that the prob lem of in ter po la tion of data or functions can be gathered in the same math e mat ical scheme when we develop the concept of functional analysis with covariant and con- travariant manifolds $\widetilde{\phi}_{n}$ and $\widetilde{\phi}^{n}$. This kind of manifold re cently found and ap plied in the field of dynamics (Urrutia, 1992a and 1992b) sets up the basis for a generalized functional analysis with skew manifolds. We note that in some references (Urrutia, 1998) and (Bowen et al., 1976) at ten tion is fo cused on one man i fold unand one dual man i fold $\mathrm{v}^{\mathrm{n}}$ which are biorthogonal and are asso ciated to a nonsymmetric trans for ma tion ma trix A. For a sym met ric ma trix both spaces are equal and no new in for ma tion is given. In fact in a pre vi ous paper it has been seen that if the ma trix trans for ma tion is sym met ric we can still be able to cal cu late both man $\mathrm{i}$ folds which are identified now as un $u^{n}=\phi_{n}$ (covariant man i fold) and $\gamma^{n}=\phi^{n}$ contravariant manifold). Be sides, we will not be only con cen trated in the problem of existence, already tackled in (Urrutia, 1992a and 1992b), but rather in the di rect 
use of these mathematical tools in the solution and ap plica tion of real prob lems.

\section{Theory}

Given a set of covariant func tions $\widetilde{\phi}_{n}$ lin early in depend ent (not nec es sarily or tho go nal) in a given domain $\Omega$, there is an otherset $\widetilde{\phi}^{n}$ of contravariant functions biorthogonal to the former ones. There fore, given an ar bi trary func tion $\tilde{F}$ in the same domain $\Omega$ with norm $|\tilde{F}|$, can be decomposed in the followingman ner

$$
\tilde{F}=\sum_{n=1}^{\infty} f^{n} \widetilde{\phi}_{n}
$$

In covariant basis $\widetilde{\phi}_{\mathrm{n}}$ and contravariant components fn (sca lars) or in the form

$$
\tilde{F}=\sum_{n=1}^{\infty} f_{n} \tilde{\phi}_{n}
$$

in contravariant basis $\widetilde{\phi}^{n}$ and covariant components $\mathrm{fn}^{\mathrm{n}}$ (sca lars). There fore if equa tions (2) and (3) are available we can calculate the norm of the function (or vec tor, Urrutia, 2003) $\widetilde{F}$ in the following way

$$
\begin{gathered}
|F|^{2}=\sum_{n=1}^{\infty} f^{n} f_{n} \\
|F|=\sqrt{\sum_{n=1}^{\infty} f^{n} f_{n}}
\end{gathered}
$$

which for skew coordinate functions is the counter part and con sti tutes a gen er al iza tion of the $P y$ thag o rean the o rem used in rect an gu lar sys tems in the the ory of vec tors.

A partic u larcase oc curs, when the man ifold $\tilde{\phi}_{n}$ is or thogo nal or orthono rmal. In this case all members of the covariant man i fold $\widetilde{\phi}_{n}$ are both lin early independent and orthogonal. The contravariant basis $\tilde{\phi}^{n}$ are collinear to the functions $\tilde{\phi}_{n}$ and therefore, $\widetilde{\phi}^{n}$ is identical to $\tilde{\phi}_{n}$. In the same way $f^{n}=f_{n}$. Thus from equa tion (4) the norm of the function $\widetilde{F}$ is equal to

$$
F=\sqrt{\sum_{n=1}^{\infty} f^{n} f_{n}}=\sqrt{\sum_{n=1}^{\infty} f_{n}^{2}}
$$

for orthogonal linear manifolds. For the general case of skew coordinates, if the covariant and contravariant approximations are complete and convergent we must respect the following two equations

$$
\begin{gathered}
F=\sqrt{\sum_{n=1}^{\infty} f^{n} f_{n}} \\
F \geq \sqrt{\sum_{n=1}^{N} f^{n} f_{n}}
\end{gathered}
$$

Which are the Parseval and Bessel conditions respec tively forskewco ordinates.

\section{Norms of Skew Vectors and Continuous Functions}

Be fore em barking on further de vel op ments, we will define several operations used for discrete (vec tors, Urrutia, 2003) and continuous functions in order to cover both cases in one pre sen ta tion.

The sca lar prod uct of two vec tors $\tilde{\phi}_{n}$ and $\tilde{\phi}_{n}$ (or $\tilde{\phi}^{n}$ ) and the en ergy norm of the same vec tors with respect to the operator $K_{m n}$ are defined by the following two equa tions

$$
\begin{gathered}
<\widetilde{\phi}_{n} \widetilde{\phi}_{m,}>=\widetilde{\phi}_{n}^{\mathrm{T}} \widetilde{\phi}_{\mathrm{m}} \\
<\widetilde{\phi}_{n,} K_{\mathrm{m}} \widetilde{\phi}_{\mathrm{m}}>=\sum_{\mathrm{n}=1}^{\mathrm{N}} \sum_{\mathrm{m}=1}^{\mathrm{M}} \widetilde{\phi}_{\mathrm{n}}^{\mathrm{T}} K_{\mathrm{nm}} \widetilde{\phi}_{\mathrm{m}}
\end{gathered}
$$

where $\widetilde{\phi}_{n}$ stands for a column vector, $\widetilde{\phi}_{n}{ }^{\mathrm{T}}$ is a row vector which is the transpose of $\tilde{\phi}_{n}$ and $\boldsymbol{K}_{\mathrm{nm}}$ is a transformation matrix.

The scalar prod uct of two func tions $\widetilde{\phi}_{n}$ and $\widetilde{\phi}_{n}$ (or $\tilde{\phi}^{m}$ ) and the en ergy norm of the same func tions 
with re spect to the op era tor $\boldsymbol{K}_{\mathrm{m}}$ are de fined by the following two equations

$$
\begin{gathered}
<\tilde{\phi}_{\mathrm{n},} \tilde{\phi}_{\mathrm{m},}>=\int \Omega \tilde{\phi}_{\mathrm{n}}(\mathrm{x}) \tilde{\phi}_{\mathrm{m}}(\mathrm{x}) \mathrm{dx} \\
<\widetilde{\phi}_{\mathrm{n},} K_{\mathrm{nm}} \tilde{\phi}_{\mathrm{m}}>=\sum_{1}^{N} \sum_{1}^{M} \int \Omega \tilde{\phi}_{\mathrm{n}}(x) K_{\mathrm{nm}} \tilde{\phi}_{\mathrm{m}}(\mathrm{x}) \mathrm{dx}
\end{gathered}
$$

Despite their different aspect, equations (7) to (10) stand for an in te gra tion pro cess.

\section{Covariant and Contravariant Basis for Continuous Manifolds}

We de fine a man i fold in a do main $\Omega$ by a set of contravariant func tions $\widetilde{\phi}^{n}$ lin earlyin depend ent. A sec ond group of covariant base func tions $\widetilde{\phi}_{n}$ is defined in the same do main $\Omega$ in such a way that the sca lar prod uct be tween these two kinds of co or dinates leads us to the Kronecker symbol $\delta_{n}^{n}$ as follows

$$
\int_{\Omega} \widetilde{\phi}^{m} \widetilde{\phi}_{n} d \Omega=<\widetilde{\phi}_{n}, \widetilde{\phi}^{m} \geq \delta_{n}^{m}=\underbrace{1 m=n}_{0 m \neq n}
$$

An arbitrary function $\widetilde{F}$ can be re solved in these two man ifolds as follows

$$
\begin{gathered}
\widetilde{F}=\sum_{n=1}^{\infty} c_{n} \tilde{\phi}^{n} \\
\widetilde{F}=\sum_{n=1}^{\infty} c^{n} \widetilde{\phi}_{n}
\end{gathered}
$$

Where $C^{n}$ and $C_{n}$ stand for, the contravariant and the covariant components of $\widetilde{F}$. Any continuous function can be decomposed in covariant and contravariant basis $\widetilde{\phi}_{n}$ and $\tilde{\phi}^{n}$. So, it can be shown that when we attempt to resolve the covariant base function $\widetilde{\phi}_{\mathrm{n}}$ in covariant components the followingresult is ob tained

$$
\widetilde{\phi}_{n}=\widetilde{\phi}_{n 1} \widetilde{\phi}^{1}+\widetilde{\phi}_{n 2} \widetilde{\phi}^{2}+\widetilde{\phi}_{n 3} \widetilde{\phi}^{3}+\ldots
$$

In tensor notation

$$
\widetilde{\phi}_{\mathrm{n}}=\widetilde{\phi}_{\mathrm{nm}} \widetilde{\phi}^{\mathrm{m}}
$$

Recall that $\widetilde{\phi}_{1} \cdot \widetilde{\phi}_{n}=\widetilde{\phi}_{n} \cdot \widetilde{\phi}_{1}$ and $\phi_{m}=\phi_{m} \cdot$ In the same fashion the following decomposition is possible

$$
\widetilde{\phi}^{n}=\phi^{n m} \widetilde{\phi}_{m}
$$

In the last two equations $\phi_{m}$ and $\phi^{n m}$ are the covariant and contravariant met ric ten sors of ten sor cal cu lus. Usually, it is easy to choose an ar bi trary and com plete set of covariant base func tions. The difficult part had been to find the contravariant base functions, to overcome this difficulty we continue as follows. By hypothesis we know that the Kronecker delta func tion is ob tained when the fol low ing prod uct is per formed (Urrutia, 2003) (now an in te gral)

$$
\widetilde{\phi}_{n} \cdot \widetilde{\phi}^{m}=\delta_{n}^{m}
$$

Using the re sults (15) and (16) we find

$$
\begin{gathered}
<\phi_{n s} \widetilde{\phi}^{s}, \phi^{\mathrm{mt}} \widetilde{\phi}_{\mathrm{t}}>=\delta_{\mathrm{n}}^{\mathrm{m}} \\
\phi_{n s} \phi^{\mathrm{mt}} \delta_{\mathrm{t}}^{s}=\delta_{n}^{\mathrm{m}} \\
\phi_{n s} \phi^{\mathrm{ms}}=\delta_{n}^{\mathrm{m}}
\end{gathered}
$$

When we fix the value of $m$ and we perform the sum ma tions over the re peated index $s$, the following set of m met ric compo nents $\phi^{m}$ is obtained

$$
\begin{gathered}
\phi_{11} \phi^{\mathrm{ml}}+\phi_{12} \phi^{\mathrm{m} 2}+\phi_{13} \phi^{\mathrm{m} 3}+\phi_{14} \phi^{\mathrm{m} 4}+\ldots+=\delta_{1}^{\mathrm{m}} \\
\phi_{21} \phi^{\mathrm{ml}}+\phi_{22} \phi^{\mathrm{m} 2}+\phi_{23} \phi^{\mathrm{m} 3}+\phi_{24} \phi^{\mathrm{m} 4}+\ldots+=\delta_{2}^{\mathrm{m}} \text { (19) } \\
\phi_{31} \phi^{\mathrm{ml}}+\phi_{32} \phi^{\mathrm{m} 2}+\phi_{33} \phi^{\mathrm{m} 3}+\phi_{34} \phi^{\mathrm{m} 4}+\ldots+=\delta_{3}^{\mathrm{m}} \\
\text { etc }
\end{gathered}
$$

To illustrate the use of equation (19) let us assume that the linear manifolds $\widetilde{\phi}_{m}$ and $\widetilde{\phi}^{m}$ have 
only three components. Then equation (19) will pro vide us with three sys tems of equa tions. If in equa tion (19) we set the value of $m=1$ one set of equa tions is ob tained as fol lows with $\phi_{m n}$ known

$$
\begin{aligned}
& \phi_{11} \phi^{11}+\phi_{12} \phi^{2}+\phi_{13} \phi^{13}=1 \\
& \phi_{21} \phi^{11}+\phi_{22} \phi^{12}+\phi_{23} \phi^{13}=0 \\
& \phi_{31} \phi^{11}+\phi_{32} \phi^{12}+\phi_{33} \phi^{B}=0
\end{aligned}
$$

That in ma trix form leads to

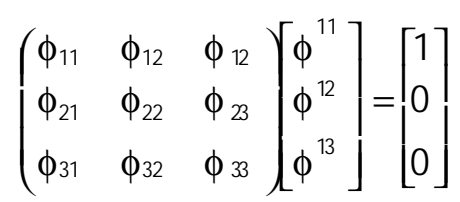

In similarfashion

$$
\left(\begin{array}{lll}
\phi_{11} & \phi_{12} & \phi_{12} \\
\phi_{21} & \phi_{22} & \phi_{23} \\
\phi_{31} & \phi_{32} & \phi_{33}
\end{array}\right)\left[\begin{array}{l}
\phi^{21} \\
\phi^{22} \\
\phi^{23}
\end{array}\right]=\left[\begin{array}{l}
0 \\
1 \\
0
\end{array}\right]
$$

And fi nally,

$$
\left(\begin{array}{lll}
\phi_{11} & \phi_{12} & \phi_{12} \\
\phi_{21} & \phi_{22} & \phi_{23} \\
\phi_{31} & \phi_{32} & \phi_{33}
\end{array}\right)\left[\begin{array}{l}
\phi^{31} \\
\phi^{32} \\
\phi^{33}
\end{array}\right]=\left[\begin{array}{l}
0 \\
0 \\
1
\end{array}\right]
$$

From this the elements $\left(\phi^{m n}\right)$ of the contravariant metric tensor ( $3 \times 3$ tensor) are calculated. With the covariant and contravariant metrics $\phi_{\mathrm{m}}$ and $\phi^{m n}$ available we can calculate the contravariant base func tions as fol lows

$$
\widetilde{\phi}^{n}=\phi^{m n} \widetilde{\phi}_{m}
$$

We can now con tinue with any fur ther anal y sis.

\section{Example 1}

Given a set of three skew covariant func tions $\tilde{\phi}_{0}=$ $1, \widetilde{\phi}_{2}=x^{2}$ and $\tilde{\phi}_{4}=x^{4}$ find the cor respond ing set of contravariant functions in the domain $-1 \leq x$ $\leq+1$. Odd powers $\left(x, x^{3}, x^{5}\right.$, etc) do not intervene be cause in a later ex am ple the $\cos (x)$ func tion (an even func tion) will be an a lyzed.

First, we have to find the elements of the covariant met rics as fol lows

$$
\begin{gathered}
<\widetilde{\phi}_{n}, \widetilde{\phi}_{m}>=\int_{-1}^{1} \widetilde{\phi}_{\mathrm{n}} \widetilde{\phi}_{\mathrm{m}}^{\mathrm{dx}}=\phi_{\mathrm{nm}} \\
\therefore \phi_{00}=\int_{-1}^{1}(1)^{2} \mathrm{dx}=2 \\
\phi_{02}=\int_{-1}^{1}(1) \mathrm{x}^{2} \mathrm{dx}=2 / 3
\end{gathered}
$$

In similar fashion we find the rest of the elements to ob tain

$$
\phi_{m n}=\left(\begin{array}{ccc}
2 & 2 / 3 & 2 / 5 \\
2 / 3 & 2 / 5 & 2 / 7 \\
2 / 5 & 2 / 7 & 2 / 9
\end{array}\right)
$$

From equation (19) we find the following equation

$$
\left(\begin{array}{ccc}
2 & 2 / 3 & 2 / 5 \\
2 / 3 & 2 / 5 & 2 / 7 \\
2 / 5 & 2 / 7 & 2 / 9
\end{array}\right)\left[\begin{array}{c}
\phi^{00} \\
\phi^{02} \\
\phi^{04}
\end{array}\right]=\left(\begin{array}{l}
1 \\
0 \\
0
\end{array}\right)
$$

From where, we ob tain the first row of the metrics matrix $\phi \mathrm{m}$. With a similar procedure we get two more equa tions and the rest of the elements of $\phi^{\mathrm{mn}}$

$$
\phi^{m}=\left(\begin{array}{ccc}
17578 & -8.2031 & 73828 \\
-8.2031 & 68.9063 & -73.8281 \\
7.3828 & -73.8281 & 861328
\end{array}\right)
$$

With the metric elements $\widetilde{\phi}^{\text {mn }}$ we can get the contravariant basis $\widetilde{\phi}^{\text {n }}$ from equa tion (16)

$$
\begin{aligned}
& \tilde{\phi}^{0}=17578-8.2031\left(x^{2}\right)+7.3828\left(x^{4}\right) \\
& \widetilde{\phi}^{0}=17578-8.2031 x^{2}+7.38284 x^{4}
\end{aligned}
$$


Similarly,

$$
\begin{gathered}
\widetilde{\phi}^{2}=-82031+68.9063 x^{2}-73.8281 x^{4} \\
\text { and } \widetilde{\phi}^{4}=7.3828-73.8281 x^{2}+861328 x^{4}
\end{gathered}
$$

The reader can ver ify that the fol low ing equa tion holds true

$$
<\widetilde{\phi}_{n}, \widetilde{\phi}^{m}>=\int_{-1}^{1} \widetilde{\phi}_{n}(x) \widetilde{\phi}^{m}(x) d x=\delta_{n}^{m}
$$

\section{Example 2, an Application to Interpolation}

Find a poly no mial ap proxi ma tion in three terms to the function $\cos (x)$ in the do main $[-1,1]$ with the following form

$$
\begin{aligned}
& \cos (x)=c^{0}+c^{2} x^{2}+c^{4} x^{4} \\
& \cos (x)=c^{0} \phi_{0}+c^{2} \phi_{2}+c^{4} \phi_{4}
\end{aligned}
$$

Use the covariant functions $\tilde{\phi}_{\mathrm{n}}$ and the contravariant functions $\widetilde{\phi}^{\text {n }}$ from example 1 . Accordingto reference (Carnaham et al ., 1969) that uses Chebyshev polyno mials the so lu tion to this problem is

$\cos (x)=0.99995795-0.49924045 x^{2}+0.03962674 x^{4}$

\section{Solution}

When we dot mul ti ply equa tion (23) by the co or dinate func tion $\widetilde{\phi}^{0}$ we get the fol lowing

$$
<\widetilde{\phi}^{0}, \cos (x)>=<\tilde{\phi}^{0},\left(c^{0} \tilde{\phi}_{0}+c^{2} \widetilde{\phi}_{2}+c^{4} \tilde{\phi}_{4}\right)>
$$

If we remember that $\left\langle\widetilde{\phi}^{n}, \widetilde{\phi}_{m}>=\delta_{m}^{n}\right.$, it is clear that the coefficient $c 0$ is obtained from the following equa tion, writ ten now in form of an in te gral

$$
\begin{gathered}
\int_{-1}^{1} \tilde{\phi}^{0} \cos (x) d x=c \\
\text { With } \tilde{\phi}^{0}=1.7578-8.2031 x^{2}+7.3828 x^{4} .
\end{gathered}
$$

When the integral is evaluated we see that $c^{0}=$ 0.999958197 . If we now per form the sca lar prod uct of equa tion (24) $\tilde{\phi}^{2}$ we will get the fol low ing

$$
<\widetilde{\phi}^{2}, \cos (x)>=<\widetilde{\phi}^{2},\left(c^{0} \widetilde{\phi}_{0}+c^{2} \widetilde{\phi}_{4}+c^{4} \widetilde{\phi}_{4}\right)>
$$

From where it is clear that the coefficient $c^{2}$ is equal to

$$
\int_{-1}^{1} \tilde{\phi}^{2} \cos (x) d x=c^{2}
$$

Where $\tilde{\phi}^{2}=-8.2031+68.9063 x^{2}-73.8281 x^{4}$. When the integral is performed we see that $c^{2}=$ -.4999309946 . In similar fashion we find $c^{4}=$ 0.039793817 . Therefore, we have that within the interval $-1 \leq x \leq 1$ the best approximation to the function $\cos (x)$ is the fol low ing

$$
\begin{gathered}
\operatorname{OS}(x)=0.999958197-0.499309946 x^{2} \\
+0.039793817 x^{4}
\end{gathered}
$$

In the basis $\tilde{\phi}_{0}=1, \widetilde{\phi}_{2}=x^{2}$ and $\widetilde{\phi}_{4}=x_{4}$. However, this ap proximation to $\cos (\mathrm{x})$ is not unique as we can re sort to the contravariant func tions $\widetilde{\phi}^{0}, \widetilde{\phi}^{2}$ and $\widetilde{\phi}^{4}$ from the first example.To make this fact clearer, we requirethe following ap proximation

$$
\cos (\mathrm{x})=c_{0} \widetilde{\phi}^{0}+c_{2} \widetilde{\phi}^{2}+c_{4} \widetilde{\phi}^{4}
$$

This is now dot mul ti plied by $\widetilde{\phi}_{0}=1$ as fol lows

$$
<\tilde{\phi}_{0}, \cos (x)>=<\widetilde{\phi}_{0},\left(c_{0} \tilde{\phi}^{0}+c_{2} \widetilde{\phi}^{2}+c_{4} \tilde{\phi}^{4}\right)>
$$

From where the fol low ing re sult is ob tained

$$
\int_{-1}^{1} \widetilde{\phi}_{0} \cos (x) d x=c_{0}
$$

When the integral is done we see that $c_{0}=$ 1.682941973. When $\tilde{\phi}_{0}$ is re placed by $\tilde{\phi}_{2}$ and by $\widetilde{\phi}_{4}$ we ob tain $c_{2}=0.478267241$ and the last co ef fi cient 
$c_{4}=0.266153329$. Therefore, the function $\cos (\mathrm{x})$ can be equally rep re sented by

$$
\begin{gathered}
\cos (x)=1682941973 \tilde{\phi}^{0}+0.478267241 \tilde{\phi}^{2} \\
+0.266153320 \tilde{\phi}^{4}
\end{gathered}
$$

With $\tilde{\phi}^{0}, \widetilde{\phi}^{2}$ y $\widetilde{\phi}^{4}$ given by the fol low ing func tions

$$
\begin{aligned}
& \widetilde{\phi}^{0}=17578-8.2031 X^{2}+7.38284 X^{4} \\
& \widetilde{\phi}^{2}={ }_{-} 8.2031+68.9063 x^{2}-73.8281 x^{4} \\
& \widetilde{\phi}^{4}=7.3828-73.8281 x^{2}+86.1328 x^{4}
\end{aligned}
$$

Equa tions (25) and (26) some how fall very close to the so lution (24) given in ref erence (Carnahamet al ., 1969). At this point we note that from the three possibleap proximations (24)to (26), theso lutions (24) and (25) that use the same covariant basis $\widetilde{\phi}_{n}$ are comparable. The problem now is to decide which of the so lu tions (24) and (25) is the best and in what sense. Any approach with given $c^{n}$ and $c_{n}$ must satisfy equations (6a) and (6b) of Parseval and Bessel for skew man i folds. In this con nec tion, Table 1 pres ents the co ef fi cients of the three approximations (24) to (26) to the function $\cos (x)$. In col umns 2, 3 and 4 are lo cated the co ef fi cients catculated according to the methods of Chebyshev and those of the pres ent paper. When for mula (6b) is applied using the coefficients of columns two and four we obtain the squared norm $|\cos (x)|^{2}=$ 1.45464763 and we get the squared root of this value we in turn obtain the norm $\cos (x)=$ 1.20608774. When the coefficients of columns three and four are equally multiplied we find that the norm of our function is $\mid \cos (x)=1.206088186$. When we find the differences of these two norms with respect to the exact value $|\cos (x)|=$ 1.206088187 (cal cu lated at the bot to $m$ of table 1 ) is 0.00000045 and 0.000000001 respectively, for the Chebyshev and the covariant approximations in the sense of norm. From this we conclude that the error of the covariant representation is 450 times smaller that the Chebyshev ap proxi mation.

As we can observeneither the Chebyshev nor the Contravariant approximations overshoot the exact norm $|\cos (x)|=1.206088187$. Therefore we can now confirm that both solutions satisfy the Bessel's inequality (6b). Up to this point we have accomplished several goals. First, we have obtained the best approximation to $\cos (x)$, in covariant basis, sec ond, we have found a new ap proxi ma tion the contravariant that al lows us to re cover the simplicity of the Pythagorean theorem, with equa tion (5), for the han dling of the con cepts of NORM and CONVERGENCE in skew manifolds. In addition we knew (Carnaham et al., 1969) that the Chebyshevap proxima tion had an errorsmaller than $4.234 \times 10^{-5}$ and now we have a new approximation the covariant with an error 450 times smaller and with a rate of convergence that satisfies the convergence laws of Parseval and Bessel. This in turn allows us to focus our attention on polynomials with powers higher than four and to ap preciate otherprob lems of nu merical analy sis.

Table 1

\begin{tabular}{lccc}
\hline & Chebyshev $^{4}$ & Contravariant & Covariant \\
\hline$a^{0}$ & 0.99995795 & 0.999970781 & 1.68294197 \\
$a^{2}$ & -0.49924045 & -0.499384548 & 0.478267252 \\
$a^{4}$ & 0.03962674 & 0.038408595 & 0.266153368 \\
$|\cos (x)|$ & 1.20608774 & 1.206088186 & \\
error & 0.00000045 & 0.000000001 & \\
\hline
\end{tabular}




\section{Higher Order Polynomial approxima- tions to $\cos (x)$ for $-1 \leq x \leq 1$}

According to what we have seen in this paper, in principle, we can obtain a covariant and a contravariant polynomials that tend to $\cos (\mathrm{x})$ in all points in the do main, i.e. we can ob tain

$$
\begin{aligned}
& \cos (x)=c^{0} \tilde{\phi}_{0}+c^{2} \tilde{\phi}_{2}+c^{4} \widetilde{\phi}_{4}+\ldots+c^{n} \widetilde{\phi}_{n} \\
& \cos (x)=c_{0} \widetilde{\phi}^{0}+c_{2} \widetilde{\phi}^{2}+c_{4} \widetilde{\phi}^{4}+\ldots+c_{n} \tilde{\phi}^{n}
\end{aligned}
$$

and the norm of $|\cos (x)|$ would be equal to $\mathrm{c}^{0} \mathrm{c}_{0}$ $+c^{2} c_{2}+c^{4} c_{4}+\ldots .+c^{n} c_{n}$ when $n \rightarrow \infty$. How ever, as we in crease the order of the matrices $\phi_{m n}$ and $\phi m$ we note that the ma trix $\phi_{m n}$ has very small elements of the order of $2 /(2(i+j)-3)$ that tend to zero when $i$ and $j$ tend to in fi nite. The vari ables $i$ and $j$ stand for the i-th row and the j-th column. This prob lem will lead us to the han dling of very ill-condi tioned ma trices of the kind of the fa mous ma trices of Hilbert with el e ments of the type $1 /(i+j)$, see ref er ence (Fraleigh and Beauregard, 1990). As it is in di cated in (Fraleigh and Beauregard, 1990), for ma tri ces of order greater than $10 \times 10$ to day's comput ers ac cu racy give rise to contravariant ma tri ces (when they are cal cu lated) $\phi^{\mathrm{mn}}$ with ex tremely large num bers that will lead us to di ver gent re sults.

When we add the re sults of poly no mi als up to 10th order to the results of the polynomial of fourth order we obtain the coefficients shown in table 2. At this point some doubts arise with re spect to the values to which the coefficients $a^{n}$ tend when $n \rightarrow$ $\infty$. We immediately note that $a^{0}$ is contained between 0.999970781 and 1.000000538, a changes between -0.499384548 and -0.500019533 , $a^{4}$ between 0.039808595 and 0.41778820 , a 6 between -0.001342159 and -0.001585556 but now we see that the coefficient of the tenth polynomial does not con verge any more and it even changes its sign.

Besides, the alternating sign of the coefficients of the poly no mial of order fourth to eight is lost in the tenth order poly no mial and this warns us that from this point on -for some reason- we start having numerical instability. From reference (Forsythe et al., 1977) we might con clude that this di ver gence may be the re sult of the Faber's The 0 rem, shown in the in tro duc tion. How ever we can not ac cept it be cause we know that the fol low ing expan sion ex ists

$$
\cos (x)=10-\frac{1}{2 !} x^{2}+\frac{1}{4 !} x^{4}-\frac{1}{6 !} x^{6}+\text { etc }
$$

and whose coefficients exactly fall between the lim it ing val ues in which the co ef fi cients of poly no $\mathrm{mi}$ als of fourth to eighth de gree. The tenth de gree polyno mial starts to di verge from expan sion (27) in view of the ill conditioningof the matrix $\phi_{m n}$ as it can be seen in equation (21). Working with dou ble or higher pre ci sion we re cover some ex act ness but soon we confront divergent approximations for higher val ues of $n$ again. In table 3 we pres ent the exact first eleven sig nif i cant con- travariant co ef ficients ob tained from equa tion (27), that our in tu ition suggests must be the coefficients that we should ob tain in table 2 if we will in crease the pre ci sion of our cal cu la tions. Fol low ing a sim i larpro cedure to the one used to calculate the contravariant poly no mial (26) the covariant co ef ficients $c_{n}$ were cal cu lated and are pre sented in the third col umn of table 3 . If the co ef fi cients $a^{n}$ and $a_{n}$ of table 3 are certainly the contravariant and the covariant co ef fi cients of $\cos x$ be tween $-1 \leq x \leq 1$ then if we calculate the norm of this function using equation (4) we must satisfy Bessel's inequa- lity (6b) when $n \rightarrow \infty$ In this sense it is readily observed that in the fourth col umn of table 3 we present the ac cu mu lated norm of $\cos (x)$ when we use equation 4 . When $n=10$ the squared norm is $|\cos (x)| z=1.454648715$ (smaller than 1.454648716 ) and it is not af fected any more for the in clu sion of the rest of the elements. From this we conclude that the poly no mial (27) con verges to $\cos (\mathrm{x})$ everywhere in the do main $-1 \leq x \leq 1$ and con verges to the norm of $\cos (\mathrm{x})$ ac cord ing to the Bessel's in equal ity (6b). In order to ob serve one more ef fect of the di vergence of the different approximationsto $\cos (x)$ 
we obtained the norms of contravariant coeffi cients of table 2 and the covariant co efficients of the third column of table 3 . The different approxi ma tions to the norm of $\cos (\mathrm{x})$ are shown in the last row of table 2.

As it can be seen, the norm of the poly no mial of fourth order is 1.454648713 , the polynomial of sixth de gree has a norm of 1.454648692 (ac tu ally it starts to diverge) and up to this point there is no major objection. However, the last two columns show norms that are greater than the exact value of 1.454648716 and this is a clear violation of the Bessel's in equal ity (6b) and a proof of di ver gence.

Table 2

\begin{tabular}{|c|c|c|c|c|}
\hline \multicolumn{5}{|c|}{ CONTRAVARIANT COEFFICIENTS OF POWERS 4, 6, 8 AND 10} \\
\hline$a^{n}$ & 4 & 6 & 8 & 10 \\
\hline$a^{0}$ & 0.999970781 & 0.999999835 & 1.000000538 & 0.999997793 \\
\hline$a^{2}$ & -0.499384548 & -0.499994769 & -0.50001953 & -0.49987840 \\
\hline$a^{4}$ & 0.039808595 & 0.041638979 & 0.041778820 & 0.040454756 \\
\hline$a^{6}$ & $-\cdots---$ & -0.001342159 & -0.00158556 & 0.002279407 \\
\hline a8 & ----- & $---\cdot-$ & 0.000129896 & -0.00450388 \\
\hline$a^{10}$ & ----- & $---\cdot-$ & $-\cdots---$ & 0.002038310 \\
\hline NORM & 1.454648713 & 1.454648692 & 1.4546488824 & 1.454650073 \\
\hline
\end{tabular}

Table 3

\begin{tabular}{cccc}
\hline $\mathrm{n}$ & $\mathrm{a}^{\mathrm{n}}$ eq $(28)$ & Covariant coeffic. $a^{\mathrm{n}}$ & Norm of $\cos (\mathrm{x})$ cumulative sum $\mathrm{a}^{\mathrm{n}} \mathrm{a}_{\mathrm{n}}$ \\
\hline 0 & +1.00 & +1.682941970 & 1.682941970 \\
2 & -0.50 & 0.478267252 & 1.443808344 \\
4 & $+1 / 4 !$ & 0.266153368 & 1.454898068 \\
6 & $-1 / 6 !$ & 0.181968530 & 1.454645334 \\
8 & $+1 / 8 !$ & 0.137541095 & 1.454648745 \\
10 & $-1 / 10 !$ & 0.110289862 & 1.454648715 \\
12 & $+1 / 12 !$ & 0.091937628 & 1.454648715 \\
14 & $-1 / 14 !$ & 0.078765706 & 1.454648715 \\
16 & $+1 / 16 !$ & 0.068865056 & 1.454648715 \\
\hline
\end{tabular}




\section{Conclusions}

From example 1 it is concluded that given a sequence of covariant func tions (com plete) $\widetilde{\phi}_{\mathrm{n}}$ there exists another set of contravariant functions $\tilde{\phi}^{n}$ which is biorthogonal to the for mer one and that satisfies the Kronecker Delta function $<\widetilde{\phi}^{m}, \widetilde{\phi}_{n}>=$ $\delta_{n}^{m}$. From example 2 we saw that any polynomial approximation to any function $f(x)$ can now be tack led by using the con cept of man i fold the ory in skew co or di nates. We must be only care ful with the con ver gence anal y sis that is di rectly re lated to the precision of the computing device available. As it was ob served, the the o rem of Faber that de nies the existence of a polynomial $P_{n}(x)$ that approaches $f(x)$, everywhere, as $n \rightarrow \infty$ is not valid. The problem of divergence shown in reference (Forsythe et al., 1977) is due to the lack of precision rather than to ques tions re lated with the ex istence or non existence of a polyno mial $P_{n}(x)$ that ap proaches $\mathrm{f}(\mathrm{x})$ as $n \infty$. Theproblem of in terpolation can now be seen as analysis in skew manifolds where equations (6a) and (6b) of Parseval and Bessel can be used to guar an tee con ver gence of our ap proximatingpolyno mials. To avoid du plication of work the in ter ested reader should re view ref er ences (Urrutia, 1992a and 1992b), to get a deeper in sight in the me chan i cal and phys i cal mean ing of the manifold the ory pre sented in this paper.

\section{Future Work}

As a fol low up to the find ings of ref er ences (Urrutia, 1992a, 1992b and 1998), and of the present paper we will use the same the ory now fo cused on the solution of non lineardifferential equations. As we will see, using covariant and contravariant manifolds will al lows us to ob tain an easy and novel method of so lu tion for this kind of non lin ear prob lems.

\section{References}

Bowen R.W. and Wang C.C. (1976). Intro duc tion to Vectors and Tensors. Plenum Press., NY.
Carnaham B., Luther H.A. and Wilkes J.O. (1969). Applied Numerical Methods. John Wiley and Sons, Inc.

Flügge W. (1972). Tensor Analysis and Continuum Mechanics. Springer-Verlag, NY.

Forsythe G.E., Malcolm M.A. and Moler C.B. (1977). Computer Methods for Mathemathical Computa tions. Prentice Hall, Inc., NJ.

Fraleigh J.B. and Beauregard R.A. (1990). Algebra Lineal. Addison- Wesley Iberoamericana, SA.

Meirovitch L. (1967). Analytical Methods in Vibra tions, Macmillan Co. London, pp. 123, 22nd line.

Mikhlin S.G. (1964). Variational Methods in Math e mat ical Physics. Trans la tion from the Russian by T. Boddington. Pergamon Press.

Soedel W. (1972). Vibrations of Shells and Plates. Marcel Dekker, Inc., NY.

Urrutia-Galicia J.L. (1992). On the Exis tence of Covariant and Contravariant Modal Forms of Dynamic Analysis. Transactions CSME (CANADA), Vol. 16, No.2, pp. 201-217.

Urrutia-Galicia J.L. (1992). Una introducción sobre la existencia de formas modales covariantes y contravariantes de análisis dinámico (An Intro duc tion on the Exis tence of Covariant and Contravariant Modal Forms of Dynamic Analysis). SISMODINAMICA (USA) Rev. Internacional.

Urrutia-Galicia J.L (1998). On the Absolute Form of the Theory of Dinamics for Beams, Plates and Shells. Mitteeilungen des Instituts fuer Statik der Universitaet Hannover, Mitteilung Nr. 47-98, Deutschland (Germany).

Urrutia-Galicia J.L. (2003). La matriz inversa generalizada (el espacio contravariante) a- 1 de matrices de orden $m \times n$, con $m n$ y la solución cerrada de este problema. Revista, Ingeniería Investigación y Tecnología, Vol. IV- No. 1- enero-marzo, Facultad de Ingeniería UNAM, ISSN 1405-7743. 


\section{Semblanza del autor}

José Luis U rrutia-Galicia. Obtuvo el grado de ingeniero civil en la Facultad de Ingeniería, UNAM en 1975; asimismo, Ios grados de maestría (1979) y doctorado (1984) en la Universidad de Waterloo, en Ontario, Canadá. Es investigador del Instituto de Ingeniería, UNAM en la Coordinación de Mecánica Aplicada. Sus áreas de interés cubren: matemáticas aplicadas y mecánica teórica, análisis tensorial, estabilidad y vibraciones de sistemas discretos, vigas, placas y cascarones. Ha recibido reconocimientos como el "Premio al Mejor Artículo" de las Transacciones Canadienses de Ingeniería Mecánica (CSME) (Montreal, Canadá 1987) por el artículo "The Stability of Fluid Filled, Circular Cylindrical Pipes, part II Experimental", también le fue otorgada la "Medalla Duggan", que es la más alta distinción de la CSME (en la universidad de Toronto, Canadá, 1990) por el artículo "On the Natural Frequencies of Thin Simply Supported Cylin d rical Shells. 